\title{
SEMILLA ORIGINAL DE DOS CULTIVARES DE ARROZ CUBANOS: RESISTENCIA A Tagosodes orizicolus MUIR (SOGATA) ${ }^{1}$
}

\author{
Noraida de Jesús Pérez-León ${ }^{2}$, Rodolfo Isidro Castro-Menduiña ${ }^{2}$, María Caridad González-Cepero ${ }^{2}$, \\ Manuel Aguilar-Portero ${ }^{3}$, Osvaldo García-Roque ${ }^{4}$
}

\section{RESUMEN}

Semilla original de dos cultivares de arroz cubanos: resistencia a Tagosodes orizicolus Muir (Sogata). El objetivo del presente trabajo fue producir semilla original de dos nuevos cultivares de arroz obtenidos en Cuba con base en la selección por su resistencia al insecto Tagosodes orizicolus Muir (Sogata). El trabajo fue desarrollado en la Unidad Científico Tecnológica de Base "Los Palacios", Cuba, durante los años 2013 y 2014. Para ello se obtuvieron las categorías de semilla original y básica, las cuales partieron de la evaluación de resistencia a T. orizicolus Muir, así como caracteres agronómicos y la selección simultánea con criterios independientes; además, se consideraron las características típicas de cada cultivar. Se identificaron 37 líneas resistentes a Tagosodes y veinte de ellas mantuvieron un comportamiento uniforme para caracteres agronómicos, las que conformaron la semilla original de cada cultivar. Se obtuvo semilla básica, de los cultivares Anays LP-14 y Roana LP-15, para entregar al sistema estatal de producción y posterior utilización extensiva en la producción nacional.

Palabras clave: Oryza sativa, rendimiento agrícola de arroz, rendimiento industrial de arroz.

\begin{abstract}
Original seed of two Cuban rice cultivars: resistance to Tagosodes orizicolus Muir (Sogata). The aim of this study was to produce original seed of two new rice cultivars obtained in Cuba, based on the selection of resistance to insect Tagosodes orizicolus Muir (Sogata). The study was developed at the Scientific Technological Base Unit (UCTB) "Los Palacios", Cuba", during 2013 and 2014. To that end, original and basic seed categories was were obtained, beginning with the evaluation of resistance to $T$. orizicolus Muir, agronomic characters and simultaneous selection with independent criteria; also the typical characteristics from each cultivar were considered. Thirty seven resistant lines to Tagosodes were identified and twenty of them kept a uniform behavior for agronomic characters, conformed the original seed of each cultivar. Basic seed from cultivars Anays LP-14 and Roana LP-15 were obtained to give them to the tate production system and for the subsequent extensive use in national production.
\end{abstract}

Keywords: Oryza sativa, rice agricultural yield, rice industrial yield.

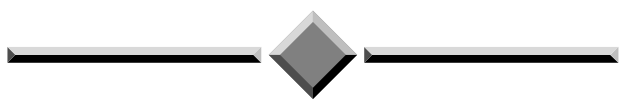

\footnotetext{
Recibido: 2 de setiembre, 2015. Aceptado: 28 de octubre, 2015. Este trabajo formó parte de la tesis de Osvaldo García Roque para la especialidad en Producción Agroindustrial del Arroz y forma parte del proyecto de investigación "Bases ambientales para la sostenibilidad alimentaria local", del Ministerio de Ciencia, Tecnología y Medio Ambiente de Cuba, implementado por el Programa de las Naciones Unidas para el Desarrollo (PNUD) y financiado por la Unión Europea.

2 Instituto Nacional de Ciencias Agrícolas (INCA). Gaveta postal 1, San José de las Lajas, Mayabeque, Cuba, CP 32 700. nory@inca.edu.cu, fofi@inca.edu.cu, mcaridad0904@gmail.com

3 Centro de Investigación y Formación Agraria (CIFA), «Las Torres Tomejil», Departamento de Arroz y Maíz, Alcalá del Río, Sevilla, España. manuel.aguilar.portero@juntadeandalucia.es

4 Universidad de Pinar del Río, Centro Universitario Municipal Los Palacios, Hermanos Saiz Montes de Oca. Cuba. osva@upr.edu.cu
} 


\section{INTRODUCCIÓN}

El programa de mejoramiento genético del cultivo del arroz cubano ha liberado para la producción, un elevado número de cultivares, poseedores de un alto potencial de rendimiento, el cual es limitado por los efectos negativos de factores climáticos, bajo aprovechamiento del período óptimo de siembra, malas prácticas culturales, deterioro de las propiedades de los suelos, afectaciones provocadas por plagas, el predominio de un solo cultivar en grandes extensiones y la calidad de la semilla (MINAG, 2012).

Hasta 1959 la utilización de semillas de calidad era prácticamente nula (Doria, 2010), desde 1974 se estableció el sistema de producción de semilla certificada, que ha estado dirigido a satisfacer la demanda del sector especializado y más recientemente del sector cooperativo campesino (Suárez et al., 2010).

Consecuencia de las limitaciones económicas de la década del 90, el sector agrícola cubano tuvo que enfrentar una reducción drástica en los insumos y sucedió un rápido deterioro de los sistemas usuales y centralizados de producción, mejoramiento y distribución de semillas. Desde que comenzó el fortalecimiento del sector cooperativo campesino, se ha tratado de solucionar el problema de la escasez de semilla certificada; sin embargo, las cantidades y calidad han sido insuficientes y los productores necesitan una mayor diversificación varietal que se adapte a las heterogéneas condiciones agroecológicas existentes (Morejón et al., 2014).

La alta capacidad de rendimiento en el campo e industria y la respuesta favorable en la resistencia a plagas, son factores esenciales que se mantienen si se cuenta con semilla de alta calidad. Además, esta debe poseer un alto porcentaje de germinación, excelente vigor y el cultivar debe ser resistente al acame y al desgrane (Ortega, 2014).

La semilla original es aquella que da origen al cultivar, con las características que el fitomejorador generó a través de un método de mejoramiento genético específico. Esta categoría constituye la base para el inicio de un programa de producción de semilla de alta calidad genética, razón por la que siempre deberá estar bajo el resguardo de la institución que la generó (Ortega, 2014).

La utilización de semilla nacional para el ciento por ciento de las siembras que se realizan en el país, constituye un logro del programa nacional de mejoramiento genético del cultivo (Pérez et al., 2012). Esto elimina su importación y los mejoradores del cultivo se sienten obligados a obtener semilla de calidad, que permita la expresión en campo de las bondades de los cultivares mejorados (García et al., 2014), además de tener en cuenta que la conservación y utilización sostenible de los recursos fitogenéticos son fundamentales para mejorar la productividad y la sostenibilidad de la agricultura, contribuyendo así a la seguridad alimentaria, el alivio de la pobreza y el mantenimiento de la diversidad biológica, al ofrecerse mayor número de cultivares mejorados.

Las pérdidas causadas por plagas representan una de las principales limitantes en el incremento de la productividad del arroz, causando un aumento significativo de los costos de producción (Vivas et al., 2009). El insecto Tagosodes es una de las máximas preocupaciones de los arroceros del continente americano por los daños económicos que ocasiona. En Cuba, desde la década del 70 en que se produjeron grandes afectaciones, en zonas arroceras del país, el programa de mejoramiento depuró gran parte del material, aplicando la selección, para integrar las líneas de la semilla original, con solo aquellas que resultaron resistentes e intermedias a Tagosodes, con lo cual se garantiza una mayor respuesta al daño del insecto, de esta manera se contrarresta su efecto nocivo sobre la producción y permite mantener el manejo integrado de la plaga (Gutiérrez et al., 2013).

Este trabajo tuvo como objetivo producir semilla original de dos nuevos cultivares de arroz obtenidos en Cuba con base en la selección por su resistencia al insecto Tagosodes orizicolus Muir (Sogata).

\section{MATERIALES Y MÉTODOS}

En la Unidad Científico Tecnológica de Base (UCTB) "Los Palacios", perteneciente al Instituto Nacional de Ciencias Agrícolas, ubicada en la llanura sur de la provincia de Pinar del Río, Cuba, durante los años 2013 y 2014 fueron llevados a cabo los trabajos de campo. Las labores fitotécnicas se realizaron según el Instructivo Técnico para el Cultivo del Arroz (MINAG, 2012) y se emplearon los cultivares Anays LP-14, de ciclo corto y Roana LP-15 de ciclo medio, obtenidos en Cuba mediante hibridaciones (Pérez et 
al., 2005; 2007). Para la obtención de semilla original (primera fase), en los estudios de regionalización se seleccionaron 100 panículas de cada cultivar. Estas fueron trilladas manualmente, envasadas en sobres pequeños, enumeradas, secadas al sol hasta alcanzar el porcentaje de humedad requerida (entre 12,5 y $13,5 \%$ ) y posteriormente enviadas al Instituto de Investigaciones de Granos para evaluación de resistencia a Tagosodes orizicolus Muir (Sogata), según la metodología que detallan García et al. (2014).

Se realizó la selección de líneas teniendo en cuenta la resistencia mostrada, según la escala de 9 grados para la evaluación del daño mecánico de T. orizicolus Muir (Sogata) en el área foliar de cultivares de arroz, propuesta por el IRRI (2002), la cual considera los grados entre 0-3 como resistentes, 5 intermedio y entre 7-9 como susceptibles.

Las líneas que resultaron resistentes fueron trasplantadas al campo (una planta por sitio), conservando la identidad de las panículas de las cuales provenían, en dos o tres surcos de $5 \mathrm{~m}$ de largo, dependiendo de la disponibilidad de plantas, con una distancia de $30 \mathrm{~cm}$ entre plantas y $40 \mathrm{~cm}$ entre surcos. En el Cuadro 1 se presentan las evaluaciones realizadas por etapas de crecimiento y desarrollo del cultivo.

Se realizó una selección simultánea sobre todos los caracteres evaluados, con criterios independientes, considerando además las características típicas de cada cultivar. Para el procesamiento de los datos obtenidos se utilizó el paquete estadístico SPPSS versión 11,0 sobre Windows y estos fueron sometidos a análisis multivariado de clasificación automática (conglomerados) (Varela, 1998). Además, se les determinó los valores máximo, mínimo, la media, el error estándar y coeficiente de variación, para cada carácter evaluado.

Las líneas que resultaron uniformes para los caracteres evaluados, fueron cosechadas y trilladas manualmente, secadas al sol hasta alcanzar entre 12,5 y $13,5 \%$ de humedad y venteadas para eliminar granos vanos y restos de tallos, y se conservaron independientes como líneas de semilla original.

Para obtener la semilla original (segunda fase), las líneas seleccionadas anteriormente se sembraron en campo y a los veinte días de germinadas se les evaluó el vigor, según la escala propuesta por el IRRI (2002), diez días después fueron trasplantadas (una planta por sitio), manteniendo la identidad de la panícula de la cual provenían, en parcelas de $4 \times 5 \mathrm{~m}^{2}$ con una distancia de $30 \mathrm{~cm}$ entre surcos y entre plantas y $40 \mathrm{~cm}$ de pasillo entre parcelas.

Además de las evaluaciones realizadas en la primera fase, también se evaluaron, en el momento de la cosecha, el rendimiento agrícola en un área de $1 \mathrm{~m}^{2}$ por parcela y el rendimiento industrial expresado en porcentaje de granos enteros en muestras de $1 \mathrm{~kg}$ de arroz cáscara. Se realizó una segunda selección simultánea sobre todos los caracteres evaluados, con criterios independientes, se consideraron las características típicas de cada cultivar. Los datos obtenidos fueron sometidos a análisis multivariado de clasificación automática (conglomerados) (Varela, 1998) y se les determinaron los valores máximo, mínimo, la media, el error estándar y coeficiente de variación para cada

Cuadro 1. Evaluaciones realizadas para la selección de líneas que conforman la semilla original de los cultivares de arroz Anays LP-14 y Roana LP-15, por etapas de crecimiento y desarrollo del cultivo. Cuba. 2013-2014.

Table 1. Evaluations conducted for the selection of lines of the original seed from rice cultivars Anays LP-14 and Roana LP-15, by crop growth and development stages. Cuba. 2013-2014.

\begin{tabular}{ll}
\hline Etapa de crecimiento y desarrollo & Evaluaciones* \\
\hline Entre los 55 y 65 días de germinado el arroz & Ahijamiento \\
Floración & Días para alcanzar el 10, 50 y 90\% de floración \\
Maduración & Acame, desgrane y la altura final \\
Cosecha & Cristalinidad (100 semillas por surco) \\
\hline
\end{tabular}

*Las evaluaciones fueron realizadas según la escala propuesta por el IRRI (2002) / The evaluations were carried out according to the scale proposed by IRRI (2002). 
carácter evaluado, mediante el paquete estadístico SPPSS versión 11,0 sobre Windows. Las líneas que se mantuvieron uniformes fueron cosechadas y trilladas manualmente, secadas al sol hasta alcanzar entre 12,5 y $13,5 \%$ de humedad y venteadas para eliminar granos vanos y restos de tallos.

Cada línea se conservó independiente conformando la semilla original de cada cultivar, y para la obtención de la semilla básica se mezclaron cantidades iguales de cada línea independiente según los procedimientos y normas para la producción de semillas de arroz propuesto por el Grupo agroindustrial de granos y el Instituto de investigaciones de granos de Cuba (GAIG - IIA, 2012) y se sembraron semilleros en áreas completamente libres de mezclas de otros cultivares y arroz rojo. Entre 25 y 30 días después de la germinación fueron trasplantados (una planta por sitio) en hileras dobles separadas a $40 \mathrm{~cm}$, para facilitar la selección negativa, y $30 \mathrm{~cm}$ entre plantas y surcos.

Durante el ciclo del cultivo se realizaron varias selecciones negativas, actividad mediante la cual se eliminaron plantas fuera de tipo, mezclas de otros cultivares o especies, plantas enfermas, débiles, lesionadas y otras arvenses (GAIG - IIA, 2012). La cosecha y la trilla se realizaron mecanizadas, los equipos e implementos utilizados estuvieron completamente limpios para evitar las mezclas. Los envases para la cosecha fueron nuevos y debidamente identificados. El secado de la semilla fue al sol, una vez alcanzado el porcentaje de humedad requerida (entre 12,5 y 13,5\%) fue clasificada para eliminar restos de cosecha y granos vanos.

La semilla básica fue inspeccionada y evaluada en campo y laboratorio por el Área de Certificación de Semillas del Centro Nacional de Sanidad Vegetal, según los criterios de la Norma Ramal Semillas de Arroz (Oryza sativa L.) - Certificación (MINAG, 2009) y fueron emitidos los certificados oficiales de calidad.

\section{RESULTADOS Y DISCUSIÓN}

En la Figura 1 se presentan los resultados del comportamiento de los cultivares de nueva introducción Anays LP-14 y Roana LP-15 frente al insecto Tagosodes orizicolus Muir (Sogata) en condiciones controladas.

Las líneas segregaron por su reacción frente a Tagosodes con rangos comprendidos entre los grados

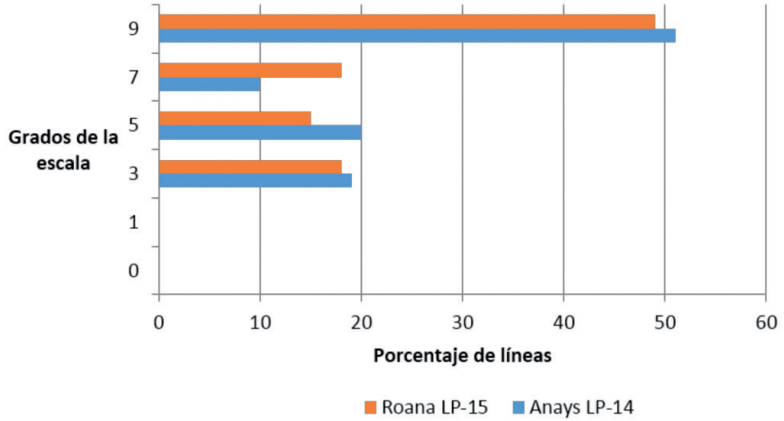

Figura 1. Porcentaje de líneas de los cultivares Anays LP-14 y Roana LP-15 de arroz, resistentes y susceptibles a Tagosodes orizicolus Muir, según la escala propuesta por el IRRI (2002), en el Invernadero del Instituto de Investigaciones de Granos, Cuba en el año 2013.

Figure 1. Percentage of lines from rice cultivars Anays LP14 and Roana LP-15, resistant and susceptible to Tagosodes orizicolus Muir, as proposed by IRRI (2002) scale, in the greenhouse of the Grain Research Institute, Cuba in 2013.

tres y nueve; es decir, se comportaron desde resistentes hasta susceptibles, pero con ausencia de las clases cero y uno, y más del $50 \%$ de las líneas de los dos cultivares fueron evaluados con los grados máximos (siete y nueve). Fueron seleccionadas diecinueve líneas resistentes de Anays LP-14 y dieciocho de Roana LP15 con grado 3 de la escala, para sembrar la primera fase de semilla original de cada cultivar.

Al evaluar el acame y desgrane en la semilla original (primera fase), el comportamiento de las líneas, dentro de cada cultivar, fue uniforme, razón por la que no se realizó selección para estos caracteres. Destaca que todas las líneas fueron resistentes al acame; al respecto se plantea que esta característica se asocia con la extensión del sistema radical, el tamaño del tallo y el espesor de su pared, con la resistencia y el espesor de la vaina, el tamaño de los entrenudos y con la altura de la planta, así como otros factores, dentro de ellos la fertilización y el manejo de la lámina de agua (Santiago et al., 2013; Alfonso, 2014).

Con respecto al desgrane, se encontraron diferencias entre los cultivares, ya que el cultivar Anays LP-14 mostró resistencia mientras que Roana LP-15 se comportó como intermedia.

Al evaluar el resto de los caracteres, sobre la base de la clasificación automática (conglomerados), 
se apreció que las diecinueve líneas resistentes del cultivar Anays LP-14, se agruparon en ocho clases (Figura 2).

Las clases cuatro, seis, siete y ocho (Cuadro 2) presentaron cuatro líneas con los valores más bajos de cristalinidad de los granos, entre 68 y $77 \%$, además coincidió en que fueron las más altas con valores entre 93,9 y 101,8 cm. La línea ubicada en la clase cinco mostró un ciclo más corto, alrededor de veinte días, y las tres líneas pertenecientes a las clases uno y tres, aunque con alturas similares a las de la clase dos, se diferencian de estas últimas por el porcentaje de granos cristalinos y los días para alcanzar la fase de floración.

Para conformar la semilla original del cultivar Anays LP-14, fueron seleccionadas solo las once líneas ubicadas en la clase dos que mostraron valores medios de doce hijos por planta, alcanzaron el 10, 50 y $90 \%$ de floración a los 93, 96 y 100 días, respectivamente, una altura de $87,4 \mathrm{~cm}$ y $90 \%$ de granos cristalinos, todo lo cual coincidió con las características del cultivar Anays LP-14, según lo informado por Pérez et al. (2005). Los coeficientes de variación (Cuadro 3) de estos caracteres fueron bajos, lo que demostró la uniformidad de las líneas.

Las dieciocho líneas resistentes del cultivar Roana LP-15 evaluadas, también en su primera fase original,

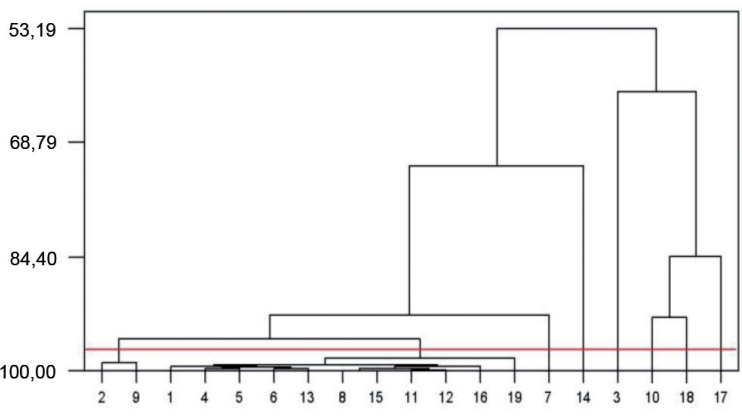

Figura 2. Dendograma obtenido a través del análisis de conglomerados realizado a la evaluación agronómica de las líneas de arroz del cultivar Anays LP-14, en la primera fase original, en la Unidad Científico Tecnológica de Base "Los Palacios", Cuba, en el año 2013.

Figure 2. Dendrogram obtained through cluster analysis performed for agronomic evaluation of rice lines from cultivar Anays LP-14, in the original first phase, at the Scientific Technological Base Unit (UCTB) "Los Palacios", Cuba, in 2013.

se agruparon en cuatro clases (Figura 3), sobre la base de la clasificación automática (conglomerados).

Los valores medios de la altura por clase (Cuadro 4) presentaron poca variación, las diferencias encontradas no superaron los $2,3 \mathrm{~cm}$, la clase cuatro

Cuadro 2. Valores medios de los caracteres evaluados en cada clase establecida sobre la base de la diversidad existente, para las líneas de arroz, del cultivar Anays LP-14 evaluadas en la primera fase original, en la Unidad Científico Tecnológica de Base "Los Palacios", Cuba, en el año 2013.

Table 2. Means values from the characters evaluated in each established class on the basis of the existent diversity, for rice lines, from cultivar Anays LP-14 evaluated in the original first phase, at the Scientific Technological Base Unit (UCTB) "Los Palacios", Cuba, in 2013.

\begin{tabular}{|c|c|c|c|c|c|c|c|}
\hline \multirow[t]{2}{*}{ Clases } & \multirow{2}{*}{$\begin{array}{l}\text { No. } \\
\text { líneas }\end{array}$} & \multirow{2}{*}{$\begin{array}{l}\text { Hijos por } \\
\text { planta }\end{array}$} & \multicolumn{3}{|c|}{ Floración } & \multirow{2}{*}{$\begin{array}{c}\text { Altura } \\
(\mathrm{cm})\end{array}$} & \multirow{2}{*}{$\begin{array}{c}\text { Granos } \\
\text { cristalinos }(\%)\end{array}$} \\
\hline & & & $10 \%$ & $50 \%$ & $90 \%$ & & \\
\hline 1 & 2 & 11 & 97 & 100 & 103 & 84,7 & 85 \\
\hline 2 & 11 & 12 & 93 & 96 & 100 & 87,4 & 90 \\
\hline 3 & 1 & 10 & 87 & 90 & 94 & 86,0 & 95 \\
\hline 4 & 1 & 11 & 98 & 101 & 104 & 93,9 & 68 \\
\hline 5 & 1 & 14 & 73 & 76 & 80 & 84,7 & 86 \\
\hline 6 & 1 & 14 & 85 & 88 & 91 & 101,8 & 77 \\
\hline 7 & 1 & 12 & 87 & 90 & 94 & 101,8 & 77 \\
\hline 8 & 1 & 13 & 77 & 80 & 83 & 94,8 & 68 \\
\hline
\end{tabular}


Cuadro 3. Variación observada en las once líneas del cultivar Anays-LP14, seleccionadas en la primera fase original, en la Unidad Científico Tecnológica de Base "Los Palacios", Cuba, en el año 2013.

Table 3. Variation observed in the eleven lines from cultivar Anays-LP14, selected in the original first phase, at the Scientific Technological Base Unit ( UCTB ) "Los Palacios", Cuba, in 2013.

\begin{tabular}{llllll}
\hline Caracteres evaluados & Min & Max & X & ESx & CV \\
\hline Ahijamiento (hijos / planta) & 11 & 14 & 12 & 0,25 & 6,83 \\
10\% de floración (días) & 90 & 94 & 93 & 0,35 & 1,26 \\
50\% de floración (días) & 93 & 97 & 96 & 0,34 & 1,17 \\
90\% de floración (días) & 97 & 101 & 100 & 0,34 & 1,13 \\
Altura (cm) & 85,7 & 89,9 & 87,4 & 0,50 & 1,91 \\
Granos cristalinos (\%) & 89 & 93 & 90 & 0,34 & 1,24 \\
\hline
\end{tabular}

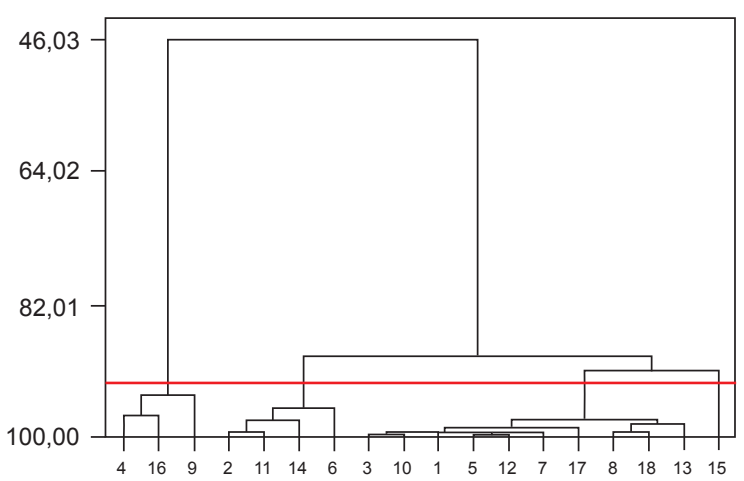

Figura 3. Dendograma obtenido a través del análisis de conglomerados realizado a la evaluación agronómica de las líneas de arroz del cultivar Roana LP-15, en la primera fase original, en la Unidad Científico Tecnológica de Base "Los Palacios", Cuba, en el año 2013.

Figure 3. Dendrogram obtained through cluster analysis performed for agronomic evaluation of rice lines from cultivar Roana LP-15, in the original first phase, at the Scientific Technological Base Unit (UCTB) "Los Palacios", Cuba, in 2013.

estuvo representada por la línea con el valor más bajo de cristalinidad de los granos (84\%) y las tres líneas ubicadas en la clase uno mostraron un ciclo de alrededor de diez a trece días más corto. Las clases dos y tres agruparon líneas con un comportamiento similar para el número de hijos por planta, altura y porcentaje de granos cristalinos, pero las cuatro líneas de la clase dos fueron cuatro días de ciclo más cortas.
Para conformar la semilla original del cultivar Roana LP-15 se seleccionaron solo las diez líneas ubicadas en la clase tres con un ahijamiento promedio de trece hijos por planta, 101, 104 y 108 días para alcanzar el 10,50 y $90 \%$ de floración, respectivamente, una altura de $96,2 \mathrm{~cm}$ y $91 \%$ de granos cristalinos, lo que coincidió con lo informado por Pérez et al. (2007). Los coeficientes de variación (Cuadro 5) determinados fueron bajos, lo que es un indicador de la homogeneidad fenotípica de las líneas seleccionadas, para los caracteres evaluados.

Se seleccionaron once líneas del cultivar Anays LP-14 y diez de Roana LP-15, las cuales fueron utilizadas para su siembra en la segunda fase. Al evaluar los caracteres número de hijos por planta, altura, días necesarios para alcanzar el 10, 50 y $90 \%$ de floración y cristalinidad de los granos (datos no mostrados), las líneas de los dos cultivares se mantuvieron con valores similares a la evaluación efectuada en la primera fase original, por lo que en este sentido no se realizó selección.

De las líneas seleccionadas en la primera fase original, solo una de Anays LP-14 mostró menor rendimiento agrícola e industrial, el resto se mostró uniforme con valores de rendimiento agrícola entre 6,9 y 7,5 t/ha para Anays LP-14 y entre 7,0 y 7,8 t/ha para Roana LP-15 (Cuadro 6). Similar comportamiento ocurrió para el rendimiento industrial con valores entre 58,2 y $60,1 \%$ de granos enteros para Anays LP-14 y entre 57,7 y $59,4 \%$ para Roana LP-15. Se tomaron en cuenta ambos caracteres evaluados para la selección 
Cuadro 4. Valores medios de los caracteres evaluados en cada clase establecida sobre la base de la diversidad existente, para las líneas de arroz del cultivar Roana LP-15, evaluadas en la primera fase original, en la Unidad Científico Tecnológica de Base "Los Palacios", Cuba, en el año 2013.

Table 4. Means values from the characters evaluated in each established class on the basis of the existent diversity, for rice lines, from cultivar Roana LP-15 evaluated in the original first phase, at the Scientific Technological Base Unit ( UCTB ) "Los Palacios", Cuba, in 2013.

\begin{tabular}{|c|c|c|c|c|c|c|c|}
\hline \multirow[b]{2}{*}{ Clases } & \multirow{2}{*}{$\begin{array}{l}\text { No. } \\
\text { líneas }\end{array}$} & \multirow{2}{*}{$\begin{array}{c}\text { Hijos por } \\
\text { planta }\end{array}$} & \multicolumn{3}{|c|}{ Floración } & \multirow{2}{*}{$\begin{array}{c}\text { Altura } \\
(\mathrm{cm})\end{array}$} & \multirow{2}{*}{$\begin{array}{c}\text { Granos } \\
\text { cristalinos }(\%)\end{array}$} \\
\hline & & & $10 \%$ & $50 \%$ & $90 \%$ & & \\
\hline 1 & 3 & 12 & 87 & 90 & 94 & 95,4 & 94 \\
\hline 2 & 4 & 12 & 97 & 100 & 104 & 94,8 & 93 \\
\hline 3 & 10 & 13 & 101 & 104 & 108 & 96,2 & 91 \\
\hline 4 & 1 & 14 & 98 & 101 & 105 & 93,9 & 84 \\
\hline
\end{tabular}

Cuadro 5. Variación observada en las diez líneas de arroz del cultivar Roana-LP15, seleccionadas en la primera fase original, en la Unidad Científico Tecnológica de Base (UCTB) "Los Palacios", Cuba, en el año 2013.

Table 5. Variation observed in the ten lines of cultivar Roana-LP15, selected in the original first phase, at the Scientific Technological Base Unit ( UCTB ) "Los Palacios", Cuba, in 2013.

\begin{tabular}{lccccc}
\hline Caracteres evaluados & Min & Max & X & ESx & CV \\
\hline Ahijamiento (hijos/ planta) & 12 & 14 & 13 & 0,25 & 6,08 \\
10\% de floración (días) & 100 & 103 & 101 & 0,34 & 1,07 \\
50\% de floración (días) & 103 & 106 & 104 & 0,34 & 1,04 \\
$90 \%$ de floración (días) & 107 & 110 & 108 & 0,37 & 1,09 \\
Altura (cm) & 95,1 & 97,3 & 96,2 & 0,24 & 0,77 \\
Granos cristalinos (\%) & 89 & 93 & 91 & 0,49 & 1,70 \\
\hline
\end{tabular}

de las diez líneas de cada cultivar para conformar sus semillas originales.

En el Cuadro 7 se presenta la producción de semilla básica obtenida de los cultivares Anays LP-14 y Roana LP-15, cuya finalidad fue la siembra en las fincas de semilla para la reproducción de las siguientes categorías: venta posterior a los productores arroceros del país y garantía de generalización. Los valores de rendimiento obtenidos se mantuvieron similares a la semilla original.

El empleo de semilla de calidad reduce el área a sembrar y la necesidad de replantar, porque está garantizado un alto porcentaje de germinación, la uniformidad en la floración facilita la cosecha de los granos, el vigor en generaciones tempranas le permite competir con las arvenses, aportan mayores rendimientos y mejora la calidad del grano al no estar contaminada con otros cultivares (Asea et al., 2010).

Los resultados obtenidos permitieron identificar líneas resistentes a Tagosodes. Las que mantuvieron un comportamiento uniforme, para caracteres agronómicos, conformaron la semilla original de cada cultivar. Se obtuvo la semilla básica para entregar al sistema estatal para la reproducción de las siguientes categorías de semilla, y de esta forma contribuir a la mejora de la diversidad del cultivo. 
Cuadro 6. Comportamiento del rendimiento agrícola e industrial de las líneas, de los cultivares Anays LP-14 y Roana LP-15, evaluadas en la segunda fase original, en la Unidad Científico Tecnológica de Base (UCTB) "Los Palacios", Cuba, en el año 2014.

Table 6. Behavior of agricultural and industrial yield of the lines, from cultivars Anays LP-14 and Roana LP-15, evaluated in the original second phase, at the Scientific Technological Base Unit (UCTB) "Los Palacios", Cuba, in 2014.

\begin{tabular}{|c|c|c|c|c|}
\hline \multirow{3}{*}{$\begin{array}{l}\text { Cultivar } \\
\text { Anays LP-14 } \\
\text { Líneas }\end{array}$} & \multicolumn{4}{|c|}{ Rendimiento } \\
\hline & \multicolumn{2}{|c|}{ Agrícola (t/ha) } & \multicolumn{2}{|c|}{ Industrial (\% de granos enteros) } \\
\hline & Seleccionadas & Descartadas & Seleccionadas & Descartadas \\
\hline Cantidad & 10 & 1 & 10 & 1 \\
\hline Valor mínimo & 6,9 & & 58,2 & \\
\hline Valor máximo & 7,5 & & 60,1 & \\
\hline $\mathrm{x}$ & 7,2 & 5,4 & 58,9 & 48,6 \\
\hline ESx & 0,1 & & 0,2 & \\
\hline $\mathrm{CV}$ & 2,8 & & 1,0 & \\
\hline Cultivar & \multicolumn{4}{|c|}{ Rendimiento } \\
\hline Roana LP-15 & \multicolumn{2}{|c|}{ Agrícola (t/ha) } & \multicolumn{2}{|c|}{ Industrial (\% de granos enteros) } \\
\hline Cantidad & \multicolumn{2}{|c|}{10} & \multicolumn{2}{|c|}{10} \\
\hline Valor mínimo & \multicolumn{2}{|c|}{7,0} & \multicolumn{2}{|c|}{57,7} \\
\hline Valor máximo & \multicolumn{2}{|c|}{7,8} & \multicolumn{2}{|c|}{59,4} \\
\hline $\mathrm{x}$ & \multicolumn{2}{|c|}{7,4} & \multicolumn{2}{|c|}{58,4} \\
\hline ESx & \multicolumn{2}{|c|}{0,1} & \multicolumn{2}{|c|}{0,2} \\
\hline $\mathrm{CV}$ & \multicolumn{2}{|c|}{4,5} & \multicolumn{2}{|c|}{1,0} \\
\hline
\end{tabular}

Cuadro 7. Producción de semilla básica de arroz de los cultivares Anays LP-14 y Roana LP-15, en la Unidad Científico Tecnológica de Base (UCTB) "Los Palacios", Cuba, en el año 2014.

Table 7. Production of basic rice seed from cultivars Anays LP-14 and Roana LP-15, at the Scientific Technological Base Unit ( UCTB ) "Los Palacios", Cuba, in the year 2014.

\begin{tabular}{lccc}
\hline & \multicolumn{3}{c}{ Rendimiento } \\
\cline { 2 - 4 } & $\begin{array}{c}\text { Agrícola } \\
\text { (t/ha) }\end{array}$ & $\begin{array}{c}\text { Industrial (\% de } \\
\text { granos enteros) }\end{array}$ & $\begin{array}{c}\text { Producción } \\
(\mathbf{k g})\end{array}$ \\
\hline Anays LP-14 & 7,3 & 59,0 & 350 \\
Roana LP-15 & 7,6 & 58,9 & 560 \\
\hline
\end{tabular}

\section{LITERATURA CITADA}

Alfonso, M. 2014. Programa de certificación de semilla de arroz. En: Primer Curso - Taller Producción de semilla de arroz. (Portuguesa), INIA - VEN. http://www. arrozrojo.info.ve/documentos/alfonzo_manuel_01.pdf (consultado 22 dic. 2014)

Asea, G., G. Onaga, N.A. Phiri, and D.K. Karanja. 2010. Rice seed production manual. National Crops Resources Research Institute (NaCRRI) and CABI Africa, Kampala, UGA.

Doria, J. 2010. Generalidades sobre las semillas: su producción, conservación y almacenamiento. Cultivos Trop. 3:74-85.

GAIG - IIA (Grupo agroindustrial de granos - Instituto de investigaciones de granos). 2012. Procedimientos y normas para la producción de semillas de arroz. Instituto de Investigaciones de Granos, La Habana, CUB.

García, O., N. Pérez, y M.C. González. 2014. Producción de semillas de arroz con alta calidad, obtenidas en Pinar del Río. Rev. Avances 16:327-338.

Gutiérrez, A., A. Ginarte, J.L. Hernández, R. Alfonso, y O. Grande. 2013. Evaluación de la resistencia al 
daño mecánico y tóxico del Tagosodes orizicolus (Muir) en variedades de arroz. Revista ACTAF. http:// www.actaf.co.cu/revistas/revista-grano/Revista\%20 en\%20PDF\% 20(Vol\%2012\%20No\%201)/Trabajo4. pdf (consultado 6 dic. 2013).

IRRI (International Rice Research Institute). 2002. Standard evaluation system for rice. International Rice Research Institute (IRRI). Manila, PHI.

MINAG (Ministerio de la Agricultura). 2009. Norma ramal semillas de arroz (Oryza sativa L.) - Certificación (NRAG/8). Ministerio de la Agricultura, La Habana, CUB.

MINAG (Ministerio de la Agricultura). 2012. Modificaciones al instructivo técnico para el cultivo del arroz. Instituto de Investigaciones de Granos, La Habana, CUB.

Morejón, R., S.H. Díaz, G.S. Díaz, N. Pérez, y D. Ipsán. 2014. Algunos aspectos del manejo de la semilla de arroz por productores del sector cooperativo campesino en dos localidades de Pinar del Río. Cultivos Trop. 31:80-85.

Ortega, R. 2014. Manual para la producción de semilla de arroz. Folleto técnico. Instituto Nacional de Investigaciones Forestales, Agrícolas y Pecuarias, MEX.

Pérez, N., M.C. González, R.I. Castro, R.M. Cárdenas, E. Cristo, y H. Díaz. 2005. INCA LP-14: primera variedad de arroz obtenida en Cuba por cultivo in vitro de anteras de híbridos. Cultivos Trop. 26(2):55.
Pérez, N., M.C. González, R.I. Castro, R.M. Cárdenas, H. Díaz, y E. Cristo. 2007. INCA LP- 11 e INCA LP-15: Nuevas variedades de arroz para las condiciones de Cuba. Cultivos Trop. 28(4):67.

Pérez, N., M.C. González, R.I. Castro, E. Cristo, R.M. Cárdenas, H. Díaz, E. Díaz, y D. Trujillo. 2012. Impacto del Programa de Mejoramiento Genético del Arroz en la producción arrocera nacional. Nueva Empresa 8(1):60-63.

Santiago, R., R. Ruz, y O. García. 2013. Evaluación de ocho variedades de arroz (Oryza sativa L.) en el municipio Manatí, provincia Las Tunas. Innov. Tecnol. 19 (Número esp.):1-10.

Suárez, E., L.E. Rivero, F. González, Y. Kunihiro, y M. Shiraishi. 2010. Manual de producción de semilla para el arroz popular. Instituto de Investigaciones de Granos-Agencia de Cooperación Internacional del Japón, La Habana, CUB.

Varela, M. 1998. Análisis multivariado de datos. Aplicación a las ciencias agrícolas. Instituto Nacional de Ciencias Agrícolas, La Habana, CUB.

Vivas, L.E., D. Astudillo, y J. Poleo. 2009. Monitoreo de Tagosodes orizicolus M. e incidencia del virus de la hoja blanca "VHB" en el cultivo de arroz en calabozo, Estado Guárico, Venezuela. Agron. Trop. 59:57-67. 
